\title{
Desempenho Motor em Hemiparéticos Após Treino de Relaxamento do Membro Superior Náo Afetado
}

\author{
Motor Performance of Stroke Patients After Biofeedback Relaxation Training of the Unaffected Upper Limb \\ Cláudia Mendes Guimarães', Joaquim Pereira Brasil-Neto², Larissa Di \\ Oliveira ${ }^{3}$, Carlos Enrique Uribe Valencia ${ }^{4}$
}

\begin{abstract}
RESUMO
Introduçáo. Estudos recentes têm demonstrado que após acidente vascular encefálico (AVC) unilateral, o déficit motor parece ser acentuado por uma atividade inibitória excessiva exercida pelo hemisfério cerebral náo afetado, que se torna hiperexcitável. Propomos o Biofeedback Eletromiográfico para treino de relaxamento do membro superior não-parético (MSNP) como uma abordagem para a redução da hiperexcitabilidade do hemisfério intacto. Essa terapia, também, melhoraria o desempenho motor do membro superior parético (MSP) após AVC. Método. Participaram nove pacientes hemiparéticos, com idade média de 55,3 anos, não deprimidos e sem déficit cognitivo. $\mathrm{O}$ desempenho motor do MSP foi avaliado pelos Testes da Prancha de Pegboard (TPP) e da Caixa e Blocos (TCB) associados ao Eletromiograma de Superfície (EMG) em quatro músculos do MSNP. Após o treinamento os testes foram reaplicados. Resultados. O desempenho do MSP foi avaliado com ANOVA para medidas repetidas, e houve significância marginal no TPP ( $\mathrm{p}=0.079)$ e no TCB $(\mathrm{p}=0,068)$. Para o MSNP, o teste "t" pareado mostrou uma diferença significativa no TPP ( $\mathrm{p}<0,001)$. Conclusáo. O Biofeedback EMG apresenta-se como uma ferramenta potencial para a redução da entrada somatossensorial do MSNP e melhora do desempenho do MSP, além de apresentar-se como uma alternativa promissora para aprimorar o desempenho do MSNP.
\end{abstract}

Unitermos. Acidente Vascular Cerebral, Biofeedback, Paresia, Extremidade Superior.

Citação. Guimarães CM, Brasil-Neto JP, Di Oliveira L, Valencia CEU. Desempenho Motor em Hemiparéticos Após Treino de Relaxamento do Membro Superior Não Afetado.

\begin{abstract}
Introduction. Recent evidence has shown that the motor deficit after unilateral stroke may be compounded by excessive transcallosal inhibition from a hyperexcitable intact cerebral hemisphere. We hypothesize that EMG biofeedback relaxation training (EBRT) of the non-paretic upper limb (NPUL) might be able to decrease the excitability of the intact hemisphere, resulting in improvement in motor performance of the paretic upper limb (PUL) after stroke. Method. Nine hemiparetic patients were enrolled in the study, with a mean age of 55.3, without depression or cognitive deficits. For the evaluation of the PUL we have used the Purdue Pegboard Test (PPT) and the Box and Blocks Test (BBT) in association with surface EMG in several muscles of the NPUL. Patients were retested after biofeedback training. Results. Performance of the PUL was evaluated by a repeated measures ANOVA, and there was a marginal statistical significance in the PPT $(\mathrm{p}=0.079)$ and in the BBT $(\mathrm{p}=0.068)$. For the NPUL, the paired T-Test showed a significant difference in the PPT $(\mathrm{p}<0.001)$. Conclusion. EBRT is a potential tool for reducing somatosensory input to the NPUL, resulting in better performance of the PUL. In addition, the same procedure proved to be a promising alternative for improving the performance of the NPUL.
\end{abstract}

Keywords. Stroke, Biofeedback, Paresis, Upper Extremity.

Citation. Guimarães CM, Brasil-Neto JP, Di Oliveira L, Valencia CEU. Motor Performance of Stroke Patients After Biofeedback Relaxation Training of the Unaffected Upper Limb.

\footnotetext{
Trabalho realizado na Clínica-Escola de Fisioterapia da Escola Superior de Educaçáo Física e Fisioterapia do Estado de Goiás (ESEFFEGO) - Universidade Estadual de Goiás, Goiânia-GO, Brasil.

1. Fisioterapeuta, Mestre em Ciências da Saúde/ UnB, Hospital das Clínicas da Universidade Federal de Goiás, Goiânia-GO, Brasil.

2. Neurofisiologia, Pós-Doutorado / National Institutes of Health, Laboratório de Neurociências e Comportamento - UnB, Brasília-DF, Brasil.

3. Fisioterapeuta, Mestre em Ciências Médicas/Unb, Hospital Geral de Goiás (HGG), Departamento de Fisioterapia, Goiânia-GO, Brasil.

4. Médico, Mestre em Ciências da Saúde/UnB, Universidade de Brasília - Laboratório de Neurociências e Comportamento, Brasília-DF, Brasil.
}

Endereço para correspondência: Joaquim P Brasil-Neto Universidade de Brasília, Instituto de Biologia Departamento de Ciências Fisiológicas Laboratório de Neurociências e Comportamento Campus Darcy Ribeiro CEP 70910-900, Brasília-DF, Brasil. E-mail: jbrasil@unb.br

Relato de Caso Recebido em: 01/04/10 Aceito em: 16/09/10 Conflito de interesses: não 


\section{INTRODUÇÃO}

Pesquisas recentes têm demonstrado que após a lesão da área motora de um hemisfério cerebral, uma excessiva inibição transcalosa a partir do hemisfério cerebral nãolesado contribui para os déficits motores do paciente ${ }^{1,2}$. Outros trabalhos têm demonstrado déficits motores sutis também no membro náo-parético desses indivíduos, como por exemplo, fraqueza muscular no lado ipsilateral à lesão ${ }^{3}$. Também foram descritos déficits significativos no desempenho do MSNP de pacientes idosos com AVC quando comparados com população sadia da mesma faixa etária ${ }^{4}$. Desse modo, esses achados têm contribuído para o desenvolvimento de estratégias para a reabilitação dos indivíduos acometidos por AVC.

$\mathrm{Na}$ atualidade, várias abordagens constituem ferramentas reconhecidamente eficazes para a melhora do desempenho motor após uma lesão cerebral. Entre elas podemos citar as que objetivam o aumento da entrada somatossensorial da mão parética, a redução desta na mão não-afetada ou em áreas proximais do membro parético, ou o uso de medicamentos que influenciam os neurotransmissores adrenérgicos ou dopaminérgicos. Outras estratégias, mais recentes, visam promover a regulação da excitabilidade cortical através da estimulação magnética transcraniana ou pela estimulação transcraniana com corrente direta ${ }^{1,5,6}$. Entretanto, algumas delas ainda não estão largamente disponibilizadas, ou ainda, sofrem com a falta de adesão do paciente, por serem consideradas desconfortáveis.

Portanto, neste trabalho foi proposta uma medida alternativa para promover a reduçáo da entrada somatossensorial do MSNP, denominada treino de relaxamento com Biofeedback Eletromiográfico (EMG). Acreditamos que essa técnica poderia ser uma ferramenta valiosa para modular a inibição inter-hemisférica e potencialmente melhorar o desempenho motor do MSP em sujeitos com AVC.

\section{MÉTODO}

\section{Amostra}

A amostra foi composta por nove pacientes, de ambos os sexos, com idades entre 33 e 66 anos (55,3 \pm 10,9), diagnóstico de hemiparesia espástica secundária a AVC, na fase estável (6 a 36 meses pós-lesão), sem déficit cognitivo, não deprimidos, com capacidade de realizar movimentos de pinça fina e grosseira, que apresentavam uma ativação mioelétrica no membro superior não-parético $\geq 100 \mu \mathrm{V}$ durante os testes de desempenho motor e participantes do programa de reabilitação neurológica de uma Clínica-Escola de Fisioterapia (Tabela 1). Este estudo foi aprovado pelo comitê de ética em pesquisa envolvendo seres humanos (CEP) da Faculdade de Ciências da Saúde da Universidade de Brasília sob o registro 029/2007. Previamente, os pacientes assinaram o termo de consentimento livre e esclarecido para participação em pesquisa.

A localização da lesão foi determinada por ressonância nuclear magnética ou tomografia computadorizada do crânio. Sendo assim, foram encontradas lesôes

Tabela 1

Perfil da amostra

\begin{tabular}{|c|c|c|c|c|c|c|c|c|c|c|c|c|}
\hline Pte & Id. & S. & Diag. & Paresia & $\begin{array}{l}\text { Tempo } \\
\text { (meses) }\end{array}$ & MM & EMA & Edinburg & Dominância & $\begin{array}{c}\text { EFM } \\
\text { (MS) }\end{array}$ & $\begin{array}{l}\text { Dano } \\
\text { motor }\end{array}$ & EGD \\
\hline 1 & 47 & M & AVCI & $\mathrm{E}$ & 18 & 29 & $1+$ & 36 & Destro & 96 & Leve & 2 \\
\hline 2 & 63 & F & AVCI & E & 24 & 24 & 2 & 13 & Sinistro & 95 & Moderado & 10 \\
\hline 3 & 55 & M & AVCI & E & 36 & 26 & $1+$ & 36 & Destro & 96 & Leve & 5 \\
\hline 4 & 33 & M & AVCI & $\mathrm{D}$ & 13 & 30 & 1 & 30 & Destro & 91 & Moderado & 10 \\
\hline 5 & 65 & M & AVCI & E & 6 & 24 & 3 & 36 & Destro & 69 & Marcante & 6 \\
\hline 6 & 65 & M & $\mathrm{AVCH}$ & $\mathrm{D}$ & 19,4 & 26 & 2 & 26 & Destro & 88 & Moderado & 3 \\
\hline 7 & 66 & M & AVCI & $\mathrm{D}$ & 11,4 & 25 & 2 & 34 & Destro & 85 & Moderado & 6 \\
\hline 8 & 50 & M & $\mathrm{AVCH}$ & $\mathrm{D}$ & 23 & 30 & $1+$ & 12 & Sinistro & 83 & Moderado & 10 \\
\hline
\end{tabular}

Pte: paciente; Id: Idade (anos); S: Sexo; M: masculino; F: feminino; Diag: Diagnóstico; AVC: Acidente Vascular Cerebral; AVCI: Acidente Vascular Cerebral isquêmico; AVCH: Acidente Vascular Cerebral hemorrágico; E: esquerdo; D: direito; Tempo em meses; MM: Mini-Mental; EMA: Escala Modificada de Ashworth; EFM (MS): Escala de Fugl-Meyer (Membro Superior); EGD: Escala Geriátrica de Depressão ( $>10$ pontos = suspeita de depressão). 
subcorticais em 5 pacientes, corticais em 3 e córtico-subcorticais em um.

Como critérios de exclusão foram considerados: a presença de déficit cognitivo detectada através do MiniExame do Estado Mental ("Mini-Mental") 7,8, escore da Escala Geriátrica de Depressão 9 sugestivo de depressão, uso de medicação que afetasse o desempenho motor e, finalmente, valores de EMGS inferiores a $100 \mu \mathrm{V}$.

O comprometimento motor foi avaliado através da Escala de Avaliação Funcional de Fugl-Meyer ${ }^{10,11}$ e a determinação da dominância manual foi feita através do Inventário de Edinburgh ${ }^{12}$.

\section{Procedimento}

O estudo foi realizado em três etapas. Durante a primeira fase foi realizada uma avaliação geral com exame físico, avaliação do tônus muscular através da Escala Modificada de Ashworth e do comprometimento motor pela Escala de Fugl-Meyer, aplicação dos testes de MiniMental, Escala Geriátrica de Depressão e os testes de desempenho motor - Teste da Prancha de Pegboard e da Caixa e Blocos - simultâneos ao registro eletromiográfico do MSNP. Para tanto, foram monitorados os seguintes músculos: deltóide (fibras médias), bíceps braquial (porção longa), flexor superficial do carpo e extensor comum dos dedos. Quando a atividade eletromiográfica era maior que $100 \mu \mathrm{V}$ em qualquer um desses músculos durante a utilização do MSP, evidenciando uma certa atividade "em espelho" no MSNP, procedia-se à segunda etapa do estudo, o treino de relaxamento do MSNP com biofeedback EMG. Na última etapa, os testes de desempenho motor foram repetidos, simultaneamente aos registros EMGS, em duas condições: Final Sem Solicitação de Relaxamento (Final SSR) e Final com Solicitação de Relaxamento (Final CSR). O MSNP realizou os mesmos testes, todavia sem registro EMGS.

\section{Teste da Prancha de Pegboard}

O Teste da Prancha de Pegboard é um teste validado e utilizado em uma série de trabalhos científicos onde se deseja avaliar a coordenação e destreza manual fina. Ele consiste de uma prancha que em seu topo possui quatro recipientes localizados na horizontal e abaixo deles, na vertical, duas fileiras centrais com 25 buracos pequenos.
Os dois recipientes mais externos possuem 25 pinos cada, o recipiente localizado imediatamente à esquerda do centro contém 45 discos, enquanto que o recipiente situado à direita do centro possui 20 argolas. A realização do teste é possível através de cinco formas de testagem, denominadas como subtestes: 1) apenas com a mão direita; 2) apenas com a mão esquerda; 3) ambas as mãos; 4) mão direita + mão esquerda + ambas as mãos; 5) reunião (as duas mãos trabalham simultaneamente para sobrepor os quatro objetos). O tempo de desempenho é de 30 segundos para os subtestes um a quatro e de 60 segundos para o subteste cinco. A pontuação é feita do seguinte modo: A quantidade total de pinos encaixada para os subtestes um e dois; para o teste três a pontuação se dá segundo a quantidade de pares de pinos encaixada nos espaços; o subteste quatro consiste na média matemática desses três resultados; enfim a pontuação do subteste cinco é a quantidade de pinos, discos e argolas encaixados durante 60 segundos ${ }^{13}$.

\section{Teste da Caixa e Blocos}

Desenvolvido originalmente para avaliar a destreza manual grosseira de adultos com paralisia cerebral, atualmente é usado predominantemente por terapeutas ocupacionais. O teste é constituído por uma caixa repartida em seu centro, criando dois lados exatamente iguais. Uma quantidade de pequenos blocos de madeira é colocada em apenas um lado da caixa. O indivíduo avaliado deve usar a mão dominante para pegar um bloco de cada vez, transportá-lo sobre a repartição e colocá-lo do outro lado da caixa. O tempo do teste é de 60 segundos, e seráo computados o número de blocos colocados no lado oposto da caixa. Em seguida o teste é repetido utilizando dessa vez a mão não-dominante para transportar os blocos ${ }^{14}$.

\section{Treino de Relaxamento com Biofeedback EMG}

Para tal procedimento foi utilizado o equipamento Miotol $400^{\circ}$ da Miotec ${ }^{\oplus}$. O treino de biofeedback EMG foi possibilitado pelo uso do software Biotrainer $2.0^{\circ}$ da mesma empresa.

Foram realizadas quatro sessôes de treino de relaxamento com duração de aproximadamente uma hora. $\mathrm{O}$ intervalo entre as sessóes variou de três a duas vezes por semana, visto que os sujeitos participavam do estudo no 
mesmo dia do seu tratamento fisioterapêutico.

Em cada uma dessas sessões foi treinado um músculo individualmente, na seguinte ordem:

- Sessão 1 - Deltóide (fibras médias);

- Sessão 2 - Bíceps Braquial (porção longa);

- Sessão 3 - Flexor Superficial do Carpo;

- Sessão 4 - Extensor Comum dos Dedos.

O protocolo de relaxamento do MSNP foi acompanhado da realização, pelo MSP, de atividades motoras grosseiras e finas, através da utilização de brinquedos pedagógicos como o Encaixe de Blocos, a Torre Inteligente, e a colocação de palitos dentro de um frasco. Tais testes são de uso corrente no ambiente fisioterapêutico para o treino das habilidades supracitadas. Em adição, essas atividades foram propositadamente escolhidas por serem diversas dos testes de Pegboard e da Caixa e Blocos, visando evitar o aprendizado destes últimos.

Simultaneamente à execução dessas atividades pelo MSP, cada músculo do MSNP era monitorado e sua respectiva atividade elétrica podia ser visualizada por uma linha contínua na tela de um computador. $\mathrm{O}$ Biotrainer $^{\odot}$ permite a criação de um protocolo em que é traçada uma linha limite de atividade eletromiográfica a ser atingida. Em nosso estudo essa linha foi utilizada como limite máximo permitido, sendo associada a esse uma música com cunho de relaxamento. Assim, além da informação visual, quando a linha da atividade eletromiográfica ultrapassava esse limite a música era interrompida, fornecendo uma informação auditiva ao paciente. $\mathrm{Na}$ construção do protocolo o limite dessa linha foi delimitado individualmente segundo o valor médio do sinal EMG bruto de cada músculo registrado na coleta inicial.

Durante a sessão de treino de relaxamento do MSNP cada atividade motora era realizada separadamente e na mesma sequência para todos os pacientes. Inicialmente o paciente realizava a tarefa motora utilizando a sua mão parética sem qualquer interferência do fisioterapeuta. Em seguida lhe era mostrado o registro da tarefa realizada, apontadas as correçóes necessárias e então traçados os objetivos para a redução dos valores da atividade elétrica registrada. A tarefa motora era então repetida até que esse objetivo fosse alcançado. Em seguida era iniciada a próxima tarefa motora e tais procedimentos foram repe- tidos em todas as atividades motoras do protocolo e em todas as sessōes subsequentes.

Em referência à aplicação da terapêutica com Biofeedback, até a atualidade, a literatura não apresentou nenhum protocolo específico para o relaxamento do membro não-parético, exceto aquele que aborda o relaxamento da musculatura antagonista simultaneamente à contração dos músculos agonistas, com o intuito de controle da espasticidade. Desse modo, fez-se mister a construção de um protocolo específico às necessidades desse estudo e que se aproximasse à realidade da clínica fisioterapêutica. Inicialmente acreditamos que uma única sessão para cada músculo seria suficiente para que fossem alcançados os objetivos de relaxamento almejados. Consideramos ainda que, como nossos sujeitos possuíam comprometimento motor classificado entre leve e moderado, não apresentariam grandes variaçóes na graduação da espasticidade no MSP, fator esse que acarretaria maiores dificuldades para a realização do protocolo.

\section{Analise Estatística}

A análise dos dados foi realizada por um investigador cego quanto ao protocolo de intervenção e para tal procedimento foi utilizado o software SPSS versão 13.0. Uma ANOVA de Medidas Repetidas incluindo o fator Condição (Inicial, Final SSR e Final CSR) foi empregada para a análise dos resultados do desempenho com o MSP em cada teste (Pegboard e Caixa e Blocos). O critério de esfericidade foi cumprido em todas as análises e não houve necessidade de correçấo dos graus de liberdade dentro do modelo. A análise do desempenho com o MSNP foi feita mediante o teste t-pareado (Inicial e Final SSR).

Adicionalmente, o desempenho médio da amostra usando o MSNP foi comparado com o desempenho médio esperado para o sexo, idade e dominância manual segundo os valores estabelecidos pelos estudos de validação desses testes clássicos de desempenho motor (Purdue Pegboard e Caixa e Blocos). Para isso, foi calculada a diferença entre o desempenho obtido de cada sujeito e o desempenho esperado.

Por fim, foram comparados os resultados obtidos com o MSP, nas três condições (inicial, Final SSR e Final CSR) e com o MSNP nas condiçôes Inicial e Final SSR. 
O nível de significância estatística foi estabelecido em $\mathrm{p}<0,05$ para todas as análises.

\section{RESULTADOS}

\section{Membro Superior Parético}

Os valores médios do MSP para o Teste da Prancha de Pegboard foram os seguintes: Fase Inicial: 4,78 pinos/30s; Fase Final SSR: 5,56 pinos/30s; e Fase CSR: 6 pinos/30s (Gráfico 1). Desse modo, o desempenho no Teste da Prancha de Pegboard utilizando o MSP melhorou, mas não apresentou diferenças significantes entre as condições Inicial, Final SSR e Final CSR (Mauchly's $\left.\mathrm{W}=0,954, \mathrm{p}=0,848 ; \mathrm{F}_{2,16}=2,988, \mathrm{p}=0,079\right)$.

PEGBOARD
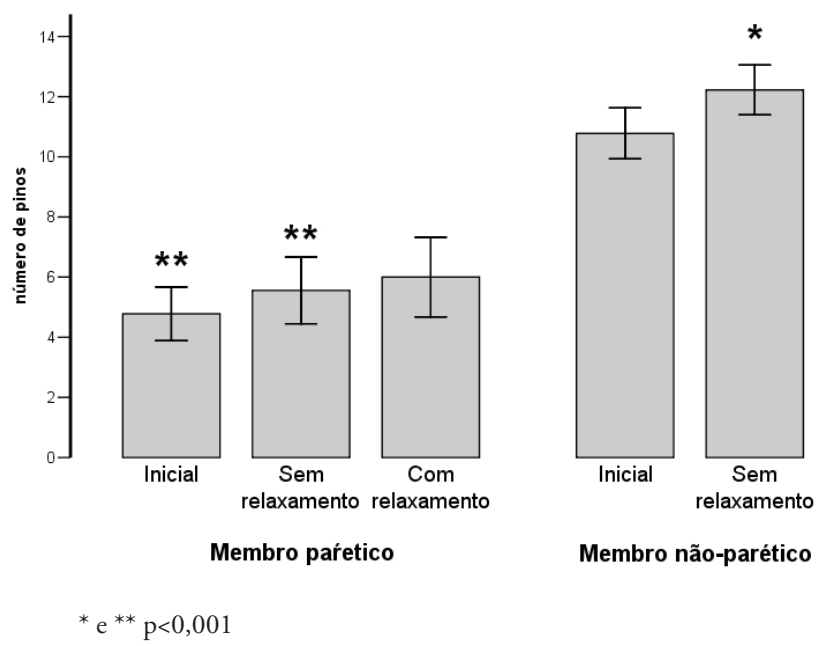

Gráfico 1. Resultados do Teste da Prancha de Pegboard para o membro superior parético e membro superior não-parético entre as condições Inicial e Final Sem Solicitação de Relaxamento.

O desempenho do MSP no Teste da Caixa e Blocos apresentou os seguintes valores médios: Fase Inicial: 34,67 blocos/min; Fase Final SSR: 36,89 blocos/min; e Fase Final CSR: 39,56 blocos/min (Gráfico 2). Apesar do aumento do número de blocos movidos por minuto, não houve diferença significante nos resultados desse teste utilizando o MSP entre as três condições (Mauchly's $\mathrm{W}=0,481, \mathrm{p}=0,077 ; \mathrm{F} 2,16=3,202, \mathrm{p}=0,068)$.

\section{Membro Superior Náo-Parético}

Os testes de destreza manual para o MSNP foram realizados em duas coletas: Inicial e Final SSR. Foram incluídos na análise os valores referentes à validação de cada teste, ajustados para o sexo, idade e dominância manual ${ }^{13,14}$. Esses valores foram coletivamente denominados "desempenho padrão".

Os resultados da análise do desempenho obtidos com o MSNP demonstraram que todos os sujeitos obtiveram resultados piores quando comparados ao desempenho padrão, tanto para o teste da Prancha de Pegboard (Gráfico 3) quanto para o da Caixa e Blocos (Gráfico 4) e em ambas as condiçóes Inicial e Final SSR (Pegboard: Inicial: $t=-7,060, p<0,001$; Final SSR: $t=-5,349, p=0,001$; Caixa e Blocos: Inicial: $\mathrm{t}=-6,308, \mathrm{p}<0,001$; Final SSR: $\mathrm{t}=-$ 4,733, $\mathrm{p}=0,001)$.

O desempenho dos sujeitos utilizando o MSNP apresentou pontuações maiores na condição Final SSR no teste de Pegboard $(\mathrm{t}=-8,222, \mathrm{p}<0,001)$ em comparação à condição Inicial (Gráfico 1). Já para o Teste da Caixa e Blocos não houve diferenças significantes entre as duas condições ( $t=-1,829, \mathrm{p}=0,105)$ (Gráfico 2).

Os resultados do MSP quando comparados ao MSNP foram piores nos dois testes e em ambas as condições (Pegboard: Inicial: t=-5,367, p=0,001.; Final SSR: $\mathrm{t}=-4,961, \mathrm{p}=0,001$. Caixas e blocos: Inicial: $\mathrm{t}=-7,003$, $\mathrm{p}<0,001$; Final SSR: $\mathrm{t}=-5,627, \mathrm{p}<0,001)$.

CAIXAS E BLOCOS

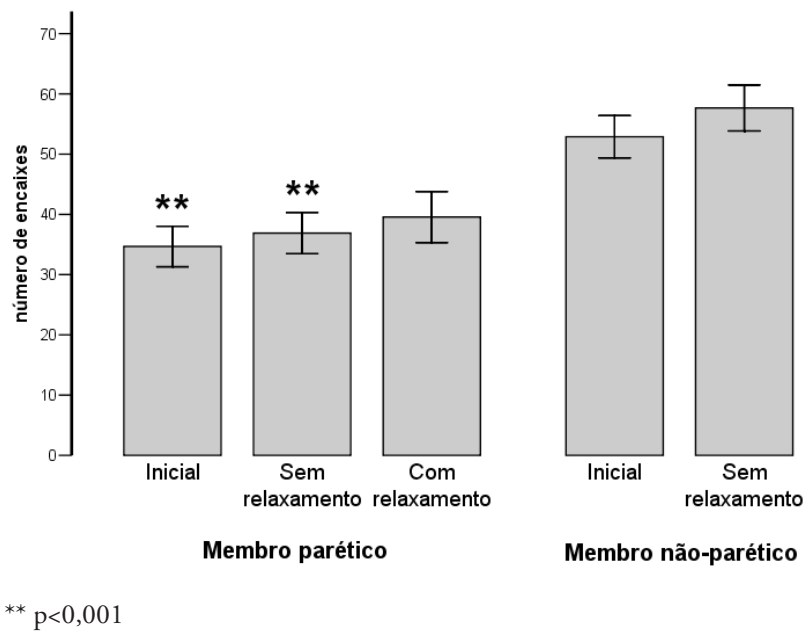

Gráfico 2. Resultados do Teste da Caixa e Blocos para o membro superior parético e membro superior não-parético.

\section{DISCUSSÃO}

Amplamente pesquisado nas décadas de 80 e 90, o Biofeedback EMG tem sido utilizado para estimular o 


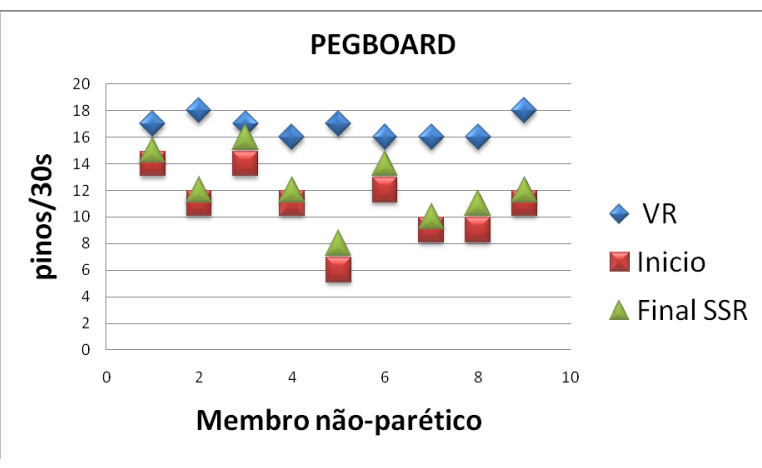

Gráfico 3. Resultados Individuais do Teste da Prancha de Pegboard para o membro superior não-parético comparados com os valores de referência (VR) e as condiçôes Inicial e Final Sem Solicitação de Relaxamento ( Final SSR).

controle motor dos músculos paralisados. Entretanto, as técnicas comumente utilizadas por esse método não contemplam o relaxamento do MSNP ${ }^{15-20}$. Portanto, este trabalho é o primeiro relato, em nosso conhecimento, que propõe o uso do biofeedback EMG para treino de relaxamento do MSNP como uma ferramenta de inibição da entrada somatossensorial e, por conseguinte, a modulação da hiperexcitabilidade do hemisfério cerebral intacto. Existem evidências de diversas fontes de que após uma lesão cortical, o hemisfério contralateral também apresenta alteraçôes plásticas. Entretanto, o cérebro humano, na tentativa de promover sua recuperação funcional, pode desenvolver alteraçôes que podem ser consideradas como mal-adaptativas ${ }^{21}$, sendo que muitos autores as descrevem tanto em relação ao membro não-parético e ao hemisfério intacto quanto ao afetado ${ }^{22}$.

Entre outros trabalhos, podemos citar um achado de evidente fraqueza muscular no lado ipsilateral da lesão em pacientes com $\mathrm{AVC}^{3}$. Por sua vez, um estudo que comparou o desempenho motor de uma população idosa pós-AVC com sujeitos normais da mesma faixa etária encontrou déficits significativos no desempenho do MSNP 4 , Escores do Teste da Caixa e Blocos também foram descritos como inferiores aos preditos para o sexo e idade da mesma população considerada normal ${ }^{23}$. Em nosso meio, ao serem estudados os déficits sensoriais da mão não-afetada em pacientes hemiparéticos, encontrou-se que a mão não-afetada desses indivíduos, além de possuir um comprometimento sensorial, quando comparada a de controles sadios, também possuía desempenho motor e força de preensão reduzidos $^{24}$. Finalmente, neste estudo os resultados dos testes

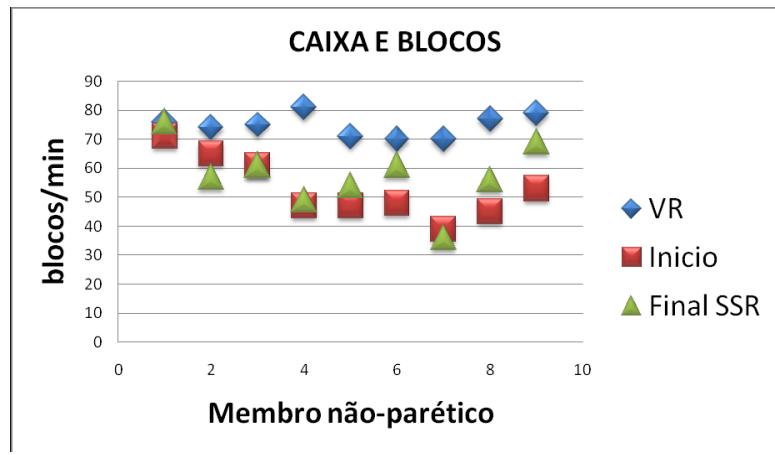

Gráfico 4. Resultados Individuais do Teste da Caixa e Blocos para o membro superior não-parético comparados com os valores de referencia (VR) e as condições Inicial e Final Sem Solicitação de Relaxamento (Final SSR).

de destreza manual para o MSNP, ajustados para o sexo, idade e dominância manual, foram inferiores aos valores considerados normais, o que confirma os relatos dos estudos acima mencionados. Assim, podemos afirmar que o membro contralateral ao distúrbio motor, largamente referido como "sadio", na verdade seria melhor denominado "membro menos afetado".

A respeito das anormalidades do equilíbrio da excitabilidade intracortical, na atualidade sabe-se que o hemisfério contralateral à lesão apresenta um excesso de atividade inibitória sobre o hemisfério infartado, influenciando sua recuperação funcional ${ }^{1,25,26}$. A ativação dos músculos do MSNP acima de $100 \mu \mathrm{V}$ encontrada nos sujeitos do presente estudo durante a realizaçáo dos testes de destreza manual, utilizando o MSP, poderia ser um indicativo de hiperatividade do hemisfério intacto. Tal condição foi eletiva para a inclusão do sujeito no protocolo de treino de relaxamento com biofeedback EMG.

Estratégias recentes de reabilitação que se utilizam do mecanismo de redução da entrada somatossensorial como uma ferramenta de modulação da excessiva inibição inter-hemisférica mostraram ganhos no desempenho motor do membro parético. Desse modo, abordagens como a anestesia cutânea e a terapia de contenção do movimento relataram êxito em sua aplicação ${ }^{27-30}$. Este estudo, por sua vez, avaliou o treino de relaxamento com biofeedback EMG como uma proposta de abordagem para a obtenção da redução da entrada somatossensorial do MSNP. Apesar dos dados apresentarem um valor limítrofe quanto à significância estatística, foi observada uma melhora no desempenho para a condição Final CSR em 
ambos os testes de desempenho motor. Isso pode sugerir que há uma tendência de que o treino de relaxamento do MSNP resulte na redução da entrada somatossensorial desse membro e, por conseguinte, na reduçáo da influência inibitória do hemisfério intacto sobre o lesado, sendo tal afirmação concordante com os achados dos estudos acima mencionados.

Um fato a ser destacado é que os achados apresentaram uma tendência de melhora para a condição Final CSR (Pegboard p= 0,079; Caixa e Blocos p=0,068), onde havia um empenho consciente em relaxar o membro estático durante a realização do movimento. Desses resultados poderiam ser aventadas as seguintes possibilidades: 1) O número da amostra foi insuficiente; 2) Não foi produzido um efeito de aprendizagem, o qual manteria a condição de relaxamento contralateral ao movimento como uma prática internalizada e constante. 3) Mesmo que o objetivo de relaxamento de cada músculo tenha sido alcançado em cada sessão correspondente, provavelmente um número maior de sessóes de treino de relaxamento com o biofeedback EMG permitiria que um efeito de retenção de aprendizado fosse melhor alcançado. Tal afirmação poderia ser comparada à importância da prática intensiva de uma tarefa utilizada na terapia de contenção do movimento, já que o relaxamento do MSNP é alcançado mediante a prática simultânea de uma tarefa motora pelo membro parético.

Em adição, encontramos que o MSNP apresentou melhora no teste da Prancha de Pegboard em relação à condição Inicial. Aparentemente, o equilíbrio da hiperexcitabilidade do hemisfério não-lesado proporcionado pelo treino de relaxamento exclusivo, já que este permaneceu em repouso durante todo o protocolo, concorreu para a melhora do desempenho motor desse membro. Como mencionado anteriormente e confirmado por nossos dados, o MSNP possui escores inferiores aos indivíduos normais nos testes de desempenho motor ${ }^{4,9,10}$. Assim, a abordagem proposta apresentou-se como uma alternativa promissora em aprimorar o desempenho do MSNP. Não se pode afirmar que tenha havido aprendizado motor do MSNP, já que foram realizadas apenas duas sessóes de testes, espaçadas no tempo; mesmo que imputássemos essa melhora a um possível aprendizado, isso indicaria uma retençẫo importante da capacidade de aprendizado mo- tor pelo membro "menos afetado", o que seria um dado importante para as estratégias de reabilitação.

Acreditamos que o número de pacientes na nossa amostra foi um fator limitante aos resultados e que a expansão desses dados resultaria em valores mais significativos. Outro importante fator é que na realidade clínica nos deparamos com a concepção terapêutica em priorizar os graus severos do comprometimento motor e a reabilitaçáo da marcha em detrimento das graduaçóes que vão do leve ao moderado, visto que os pacientes paréticos de membro superior são incomuns na rotina da clínica fisioterapêutica.

\section{CONCLUSÃO}

Os resultados sugerem que o biofeedback EMG voltado para o membro superior "menos afetado" pelo AVC pode ser uma ferramenta alternativa potencial para a redução da entrada somatossensorial do MSNP, configurando, assim, mais uma estratégia para o auxílio na reabilitação de pacientes hemiparéticos. Entretanto, consideramos necessário um estudo envolvendo um maior número de sujeitos para a sua validação, ao qual pretendemos dar continuidade em nossa instituição.

Um achado inesperado e interessante do trabalho foi que o treino de relaxamento com o biofeedback EMG mostrou-se capaz de aprimorar o desempenho do MSNP ao equilibrar a hiperexcitabilidade do hemisfério não-lesado ou ao utilizar uma considerável capacidade de aprendizado motor que possivelmente permanece intacta no hemisfério cerebral contralateral ao lesado. Novamente, estudos adicionais poderão demonstrar se um desses fatores (ou uma combinação de ambos) é responsável pela melhora do desempenho do MSNP.

\section{AGRADECIMENTOS}

À prof. Eglacy Cozenza, diretora da ESEFFEGO, pela permissão do campo de realização desse estudo.

\section{REFERÊNCIAS}

1.Ward NS, Cohen LG. Mechanisms underlying recovery of motor function after stroke. Arch Neurol 2004;61:1844-8.

http://dx.doi.org/10.1001/archneur.61.12.1844

2.Murase N, Duque J, Mazzocchio R, Cohen LG. Influence of interhemispheric 
interactions on motor function in chronic stroke. Ann Neurol 2004;55:400-9. http://dx.doi.org/10.1002/ana.10848

3.Colebatch J, Gandevia S. The distribuition of the muscular weakness in upper motor neuron lesions affecting the arm. J Physiol 1994;475:217-27.

4.Desrosiers J, Bourbonnais D, Bravo G, Roy P-M, Guay M. Performance of the "unaffected" upper extremity of elderly stroke patients. Stroke 1996;27:1564-70. http://dx.doi.org/10.1161/01.STR.27.9.1564

5.Hummel FC, Cohen LG. Non-invasive brain stimulation: a new strategy to improve neurorehabilitation after stroke? Lancet 2006;5:708-12.

http://dx.doi.org/10.1016/S1474-4422(06)70525-7

6.Bolognini N, Pascual-Leone A, Fregni F. Using non-invasive brain stimulation to augment motor training-induced plasticity. J Neuroeng Rehabil 2009;6:8. http://dx.doi.org/10.1186/1743-0003-6-8

7.Folstein M, Folstein S, McHugh P. "Mini-mental state" A practical method for grading the cognitive state of patients for the clinician. J Psychiatr Res 1975;12:189-98.

http://dx.doi.org/10.1016/0022-3956(75)90026-6

8.Bertolucci P, Brucki S, Campacci S, Juliano Y. O mini-exame do estado mental em uma população geral: impacto da escolaridade. Arq Neuropsiquiatr 1994;52:1-7.

\section{http://dx.doi.org/10.1590/S0004-282X1994000100001}

9.Yesavage J, Brink T, Rose T. Development and validation of a geriatric depression screening scale: a preliminary report. J Psychiatr Res 1983;17:37-49. http://dx.doi.org/10.1016/0022-3956(82)90033-4

10.Fugl-Meyer A, Jaasko L, Leyman I. The post-stroke hemiplegic patient: A method for evaluation of physical performance. Scand J Rehabil Med 1975;7:13-31. 11.Maki T, Quagliato E, Cacho E, Paz L, Nascimento N, Inoue M. Estudo da confiabilidade da aplicação da escala de Fugl-Meyer no Brasil. Rev Bras Fisiot 2006;10:177-83.

http://dx.doi.org/10.1590/S1413-35552006000200007

12. Oldfield R. The assessment and analysis of handedness: the Edinburgh inventory. Neuropsychologia 1971;9:97-113.

http://dx.doi.org/10.1016/0028-3932(71)90067-4

13.Tiffin J, \& Asher EJ. The Purdue Pegboard: Norms and studies of reliability and validity. J Appl Psychol, 1948;32:234-47.

http://dx.doi.org/10.1037/h0061266

14.Mathiowetz V, Volland G, Kashman N, \& Weber K. Adult norms for the Box and Block Test of manual dexterity. Am J Occup Ther, 1985;39:386-91.

15.Armagan O, Tascioglu F, Oner C. Electromyographic biofeedback in the treatment of the hemiplegic hand. Am J Phys Med Rehabil 2003;82:856-61. http://dx.doi.org/10.1097/01.PHM.0000091984.72486.E0

16.Basmajian JV. Biofeedback in rehabilitation: a review of principles and practices. Arch Phys Med Rehabil, 1981;62:469.
17.Basmajian JV, Gowland C, Brandstater ME, Swanson L, \& Trotter J. EMG feedback treatment of upper limb in hemiplegic stroke patients: a pilot study. Arch Phys Med Rehabil, 1982;63:613-6.

18. Wissel J, Ebersbach G, Gutjahr L, Dahlke F. Treating chronic hemiparesis with modified biofeedback. Arch Phys Med Rehabil, 1989;70:612-7.

19.Lonnie A Nelson. The Role of Biofeedback in Stroke Rehabilitation: Past and Future Directions. Top Stoke Rehabili 2007;14:59-66.

http://dx.doi.org/10.1310/tsr1404-59

20.Merletti R, Parker PA. Electromyography - physiology, engineering, and noninvasive applications. New Jersey: IEEE Press - John Wiley \& Sons, Inc., 2004, 494p.

http://dx.doi.org/10.1002/0471678384

21.Cohen LG. Brain stimulation in neurorehabilitation: present and future. Neurorehabil Neural Repair 2008;22:518-9.

22.Netz J, Lammers T, Homberg V. Reorganization of motor output in the non-affected hemisphere after stroke. Brain 1997;120:1579-86.

http://dx.doi.org/10.1093/brain/120.9.1579

23. Higgins J, Mayo N, Desrosiers J, Salbach N, Ahmed S. Upper-Limb function and recovery in the acute phase poststroke. J Rehabil Res Dev 2005;42:6576.

http://dx.doi.org/10.1682/JRRD.2003.10.0156

24.Brasil-Neto JP, Lima AC. Sensory deficits in the unaffected hand of hemiparetic stroke patients. Cogn Behav Neurol 2008;21:202-5.

http://dx.doi.org/10.1097/WNN.0b013e3181864a24

25.Duque J, Hummel F, Celnik P, Murase N, Mazzocchio R, Cohen LG. Transcallosal inhibition in chronic subcortical stroke. Neuroimage 2005;28:940-6. http://dx.doi.org/10.1016/j.neuroimage.2005.06.033

26.Shimizu T, Hosaki A, Hino T, Sato M, Komori T, Hirai S, et al. Motor cortical disinhibition in the unaffected hemisphere after unilateral cortical stroke. Brain, 2002;152:1896-907.

http://dx.doi.org/10.1093/brain/awf183

27.Floel A, Hummel FD, Knecht S, Cohen LG. Influence of somatosensory input on interhemispheric interactions in patients with chronic stroke. Neurorehabil Neural Repair 2008;22:477-85.

http://dx.doi.org/10.1177/1545968308316388

28.Muellbacher W, Richards C, Ziemann U, Wittenberg G, Weltz D, Boroojerdi B, et al. Improving hand function in chronic stroke. Arch Neurol 2002;59:1278-82.

http://dx.doi.org/10.1001/archneur.59.8.1278

29.Mark VM, Taub E, Morris DM. Neuroplasticity and Constraint-Induced Movement Therapy. Eur J Phys Rehabil Med 2002;42:269-84.

30.Taub E, Uswate G, Mark VW, Morris DM. The Learned nonuse phenomenon: implications for rehabilitation. Eur J Phys Rehabil Med 2006;42:241-55. 